\section{Relação entre a ocorrência de enteroparasitoses em manipuladores de alimentos e aspectos epidemiológicos em Florianópolis, Santa Catarina, Brasil}

\author{
Relationship between intestinal parasites \\ in food handlers and epidemiological factors \\ in the city of Florianópolis, Santa Catarina, Brazil
}

\author{
1 Programa de Pós-graduação \\ em Farmácia, Universidade \\ Federal de Santa Catarina, \\ Florianópolis, Brasil. \\ 2 Departamento de Análises \\ Clínicas, Universidade \\ Federal de Santa Catarina, \\ Florianópolis, Brasil. \\ Correspondência \\ G. A. Cantos \\ Departamento de Análises \\ Clínicas, Centro de Ciências \\ da Saúde, Universidade \\ Federal de Santa Catarina. \\ Campus Universitário, \\ C. P. 476, Florianópolis, SC \\ 88040-900, Brasil. \\ geny@ccs.ufsc.br
}

\begin{abstract}
The presence of intestinal parasites and epidemiological aspects were evaluated in 238 workers from a fast food company and other individuals working in street markets and farmers' produce markets in the city of Florianópolis, Santa Catarina State, Brazil. Parasitological techniques used in this study were: Lutz, Baermann-Moraes, Graham, and Faust, and parasite infection rates were $42.85 \%$ and $47.06 \%$, respectively. Socioeconomic factors such as income distribution, schooling, and occupational categories proved to be relevant in this context. Habitual daily intake of fruits and vegetables was the factor most heavily associated with infection. These data suggest that intestinal parasites are frequent among food handlers in this city and that there is a need for constant epidemiological surveillance through periodic parasitological tests and health education for this entire population segment.
\end{abstract}

Food Handling; Parasitic Diseases; Parasitological Analysis
Alexandre Costa Nolla ${ }^{1}$

Geny Aparecida Cantos ${ }^{2}$

\section{Introdução}

As doenças veiculadas por alimentos representam um importante problema de saúde pública, pois estima-se que milhões de pessoas de todo o mundo estejam acometidas por doenças transmitidas por alimentos 1 . A falta de controle higiênico de alimentos vendidos por manipuladores de alimentos constitui um importante obstáculo para implementar medidas de controle contra as parasitoses intestinais 2 . No Brasil, apesar da relevância e da atualidade do problema, são poucos os trabalhos avaliando a ocorrência de enteroparasitoses em manipuladores de alimentos. Assim, neste trabalho propôs-se delinear o perfil das enteroparasitoses entre os manipuladores de alimentos do Município de Florianópolis, Santa Catarina, Brasil.

\section{Materiais e métodos}

Esta pesquisa foi realizada com 238 indivíduos, 142 do sexo masculino e 96 do feminino, no período de janeiro 2002 a julho de 2003, na cidade de Florianópolis, os quais foram distribuídos em dois grupos: A e B. O grupo A foi constituído por 119 trabalhadores de uma empresa de alimentos (fast-food - "refeições rápidas"), com média de idade de 18 a 40 anos. O grupo B foi de 119 indivíduos que trabalhavam em feiras livres e "sacolões", com média de idade de 18 a 55 anos. 
Foi elaborado um questionário sócio-econômico padrão, aplicado a cada participante, baseado em um conjunto de questões objetivas e de múltipla escolha, descrevendo a amostra estudada. Como principais indicadores das condições sócio-econômicas dos participantes selecionaram-se as seguintes variáveis: sexo, renda familiar mensal, nível de escolaridade, número de pessoas residentes no domicílio, condições de moradia, abastecimento de água, local das refeições, destino dos dejetos, instalações sanitárias, destino do lixo e hábito de ingerir frutas e verduras ao dia.

Os métodos laboratoriais utilizados foram: Lutz, Baermann-Moraes, Graham (fita adesiva) e Faust.

A análise estatística foi baseada nos testes qui-quadrado, ANOVA e Tukey, adotando-se $\mathrm{p}<0,005$ como nível de significância 3 .

\section{Resultados e discussão}

A maioria das doenças transmitidas por alimentos está ligada às condições da matéria prima, aos maus hábitos dos manipuladores, à higienização e ao controle ambiental. Este trabalho considerou a importância dos manipuladores de alimentos como potenciais transmissores de enteroparasitoses e a possibilidade de interromper este elo na cadeia de transmissão. Pode-se notar que nas duas populações analisadas o parasitismo foi elevado, quando comparado a outros inquéritos parasitológicos 4 . No grupo A, a ocorrência de enteroparasitoses foi de $42,85 \%$ e no grupo B de $47,06 \%$. É possível atribuir este elevado parasitismo a inadequadas práticas de higiene pessoal e doméstica dos grupos estudados e ao fato de que possam ter relutância em modificar seus costumes, não dando real importância à prevenção de doenças parasitárias.

A avaliação de enteroparasitoses humanas, por meio de inquéritos parasitológicos, tem sido um parâmetro utilizado no sentido de avaliar as condições sanitárias de populações que vivem em condições precárias, no que diz respeito ao saneamento básico e às baixas condições sócio-econômicas 5. Neste trabalho, para analisar tais condições, foi solicitado aos manipuladores de alimentos que respondessem a um questionário para verificar os hábitos de higiene e as condições sanitárias do domicílio. Os resultados destas respostas estão apresentados na Tabela 1.

Com relação ao item abastecimento de água encanada e tratada e ao destino adequado dos dejetos e lixo doméstico, pode-se observar que a maioria dos indivíduos em questão, tanto parasitados como não parasitados viviam em um ambiente provido desse saneamento básico, de forma que estes dois parâmetros pouco avaliaram a condição de parasitismo. Também o hábito de comer em casa ou no trabalho não interferiu no grau de parasitismo das populações estudadas, mostrando que a contaminação fecal oral pode estar ocorrendo tanto no ambiente de trabalho como no ambiente domiciliar.

A Tabela 1 mostra também, que os fatores determinantes do elevado parasitismo foram atribuídos à menor renda familiar, ao número de pessoas residentes em cada domicílio, à escolaridade e ao hábito de ingerir verduras e frutas sem a devida higienização. Quando se levou em consideração o tamanho da família, verificou-se que os indivíduos de menor renda e menor escolaridade eram os mais parasitados. De fato, estudos mostram que a causa primária da distribuição de parasitas dentro de uma população de hospedeiros está associada a fatores de natureza demográfica 5 .

O hábito de ingerir maiores quantidades de frutas e verduras elevou o parasitismo, o que demonstrou a baixa qualidade higiênico-sanitária durante o preparo para o consumo desses alimentos nas populações estudadas. Estudos realizados no Brasil confirmaram a possibilidade de contaminação alimentar por helmintos e protozoários, devido à ingestão de hortaliças consumidas cruas, provenientes de áreas cultivadas e contaminadas por dejetos fecais 4 . Assim, é importante que haja orientação aos manipuladores de alimentos, no que diz respeito à importância da correta higienização dos alimentos, de forma a minimizar a transmissão de doenças de origem bacteriana e parasitária.

Os manipuladores de alimentos desempenham um importante papel na transmissão de doenças veiculadas por alimentos. Os riscos de contaminação dependem do grau de contato com este produto e da natureza do trabalho desempenhado. É de se esperar que grupos sociais economicamente privilegiados são pouco sujeitos a certos tipos de doenças, cuja incidência é mais elevada nos grupos economicamente desprivilegiados. De fato, nesta pesquisa pode-se notar que os gerentes e subgerentes que trabalhavam em uma empresa de alimentos foram menos parasitados que os cozinheiros, os garçons e as garçonetes (Tabela 2).

Uma das maneiras utilizadas para se garantir a qualidade higiênico-sanitária dos alimentos é a realização de programas de educação continuada para os manipuladores de alimentos, a realização semestral de exames parasitológicos desses indivíduos e o fortalecimento do 
Distribuição de alguns aspectos epidemiológicos em indivíduos parasitados e não parasitados em 238 manipuladores de alimentos (grupos A e B). Florianópolis, Santa Catarina, Brasil, 2002/2003.

\begin{tabular}{|c|c|c|c|c|c|c|c|c|}
\hline \multirow[t]{2}{*}{ Aspectos epidemiológicos } & \multicolumn{4}{|c|}{ Parasitados } & \multicolumn{4}{|c|}{ Não parasitados } \\
\hline & $n^{*}$ & $\% *$ & $n^{\star \star}$ & $\% * \star$ & $n^{*}$ & $\% *$ & $\mathrm{n}^{\star *}$ & $\%$ ** \\
\hline \multicolumn{9}{|c|}{ Renda familiar (em salários mínimos) } \\
\hline $1-3$ & 30 & 58,8 & 43 & 76,8 & 37 & 54,4 & 46 & 73,0 \\
\hline$>3$ & 21 & 41,2 & 13 & 23,2 & 31 & 45,6 & 17 & 27,0 \\
\hline \multicolumn{9}{|l|}{ Escolaridade } \\
\hline Ensino fundamental & 7 & 13,7 & 24 & 42,9 & 8 & 11,8 & 31 & 49,2 \\
\hline Ensino médio incompleto & 22 & 43,1 & 9 & 16,1 & 36 & 52,9 & 8 & 12,7 \\
\hline Ensino médio completo & 22 & 43,1 & 23 & 41,1 & 24 & 35,3 & 24 & 38,1 \\
\hline \multicolumn{9}{|l|}{ Ingestão diária de frutas } \\
\hline$<1$ & 40 & 78,4 & 18 & 32,1 & 61 & 89,7 & 26 & 41,3 \\
\hline$>1$ & 11 & 21,6 & 38 & 67,9 & 7 & 10,3 & 37 & 58,7 \\
\hline \multicolumn{9}{|l|}{ Ingestão diária de verduras } \\
\hline$<1$ & 19 & 37,3 & 20 & 35,7 & 21 & 30,9 & 24 & 38,1 \\
\hline$>1$ & 32 & 62,7 & 36 & 64,3 & 47 & 69,1 & 39 & 61,9 \\
\hline Água encanada & 47 & 98,2 & 50 & 89,3 & 64 & 94,1 & 56 & 88,9 \\
\hline Destino adequado de dejetos & 48 & 94,1 & 48 & 85,7 & 66 & 97,1 & 66 & 97,1 \\
\hline \multicolumn{9}{|l|}{ Refeições } \\
\hline Em casa & 26 & 51,0 & 30 & 56,3 & 33 & 48,5 & 26 & 41,3 \\
\hline No trabalho & 25 & 49,0 & 26 & 46,4 & 34 & 50,0 & 37 & 58,7 \\
\hline \multicolumn{9}{|l|}{ Número de pessoas residentes } \\
\hline $1-3$ & 12 & 23,5 & 29 & 51,8 & 26 & 38,2 & 39 & 61,9 \\
\hline$>3$ & 39 & 76,5 & 27 & 48,3 & 42 & 61,8 & 24 & 38,1 \\
\hline
\end{tabular}

* Grupo A: base da porcentagem = 119 entrevistados;

** Grupo B: base da porcentagem $=119$ entrevistados

sistema de vigilância sanitária para fiscalização de alimentos oferecidos pela população, incluindo uma legislação adequada.

Embora o exame clínico seja o primeiro passo para o diagnóstico das enteroparasitoses, o laboratório é essencial para que se estabeleça a espécie de parasito presente no indivíduo. Em relação às técnicas empregadas é de se considerar que cada parasitose tem a sua peculiaridade, dependendo da biologia do helminto ou protozoário a ser pesquisado. Por essa razão, não existe um método único, capaz de identificar com precisão todas as formas de parasitos. Neste trabalho foram utilizados diferentes métodos parasitológicos combinados: Lutz, Baermann-Moraes, Graham e Faust. Os resultados destas análises estão na Tabela 3.

Nota-se que a infecção por protozoários foi significativamente mais expressiva que por hel- mintos nos dois grupos analisados ( $\mathrm{p}<0,001)$, sendo que para esta análise não foram utilizados os métodos de Baermann-Moraes e Graham. Estes achados podem sugerir que a população em estudo esteja realizando uma automedicação restrita para helmintos. Porém, esta conduta além de ser contra indicada é falha quanto à abrangência no que tange as protozoonoses. Em adição, há evidências de que o uso constante de anti-helmínticos pode levar à diminuição de imunoglobulinas secretadas no intestino, predispondo certos indivíduos a condições imunopatológicas, aumentando a susceptibilidade de certas doenças como asma, diabetes do tipo I e doenças inflamatórias 6 . Além disso, trabalhos apontam que somente o tratamento com anti-helmínticos não intervém de maneira efetiva nas infecções por helmintos 7. A exemplo disso, na Região Nordeste do Bra- 


\section{Tabela 2}

Ocorrência de enteroparasitas segundo ocupação desempenhada por manipuladores de alimentos de uma empresa (grupo A). Florianópolis, Santa Catarina, Brasil, 2002/2003.

\begin{tabular}{lrrrrrr}
\hline Ocupação & \multicolumn{2}{c}{ Examinados } & \multicolumn{2}{c}{ Positivos } & \multicolumn{2}{c}{ Negativos } \\
& $n$ & \multicolumn{1}{c}{$\%$} & $\mathrm{n}$ & $\%$ & $\mathrm{n}$ & $\%$ \\
\hline Cozinheiros(as) & 61 & 51,3 & 21 & 34,4 & 40 & 65,6 \\
Garçons, garçonetes & 47 & 39,5 & 27 & 57,4 & 20 & 42,6 \\
Gerentes/subgerentes & 11 & 9,2 & 3 & 27,3 & 8 & 72,7 \\
Total & 119 & 100,0 & 51 & 42,8 & 68 & 57,2 \\
\hline
\end{tabular}

Tabela 3

Distribuição de protozoários e helmintos em 238 amostras fecais de manipuladores de alimentos, grupos A e B. Florianópolis, Santa Catarina, Brasil, 2002.

\begin{tabular}{|c|c|c|c|c|}
\hline & \multicolumn{2}{|c|}{ Grupo A } & \multicolumn{2}{|c|}{ Grupo B } \\
\hline & $\mathrm{n}$ & $\%$ & $n$ & $\%$ \\
\hline \multicolumn{5}{|l|}{ Protozoários } \\
\hline Endolimax nana & 26 & 21,8 & 26 & 21,9 \\
\hline Blastocystis hominis & 24 & 20,2 & 10 & 8,4 \\
\hline Entamoeba coli & 13 & 10,9 & 22 & 18,5 \\
\hline Giardia lamblia & 1 & 0,8 & 14 & 11,8 \\
\hline Entamoeba histolytica & 1 & 0,8 & 4 & 3,4 \\
\hline Entamoeba hartmanni & 1 & 0,8 & 0 & 0,0 \\
\hline lodamoeba butschlii & 1 & 0,8 & 2 & 1,7 \\
\hline \multicolumn{5}{|l|}{ Helmintos } \\
\hline Enterobius vermicularis & 3 & 2,5 & 8 & 6,7 \\
\hline Strongyloides stercoralis & 2 & 1,7 & 2 & 1,7 \\
\hline Ascaris lumbricoides & 1 & 0,8 & 3 & 2,5 \\
\hline Hymenolepis nana & 1 & 0,8 & 0 & 0,0 \\
\hline Total & 74 & 62,2 & 91 & 76,5 \\
\hline
\end{tabular}

sil, onde o saneamento básico é deficiente, o tratamento dos indivíduos parasitados não resulta em uma diminuição da prevalência desses parasitas 8 . Assim, o correto seria a realização do exame parasitológico, dentro dos critérios estabelecidos, assegurando uma proteção individual da saúde e bloqueando o processo de disseminação, uma vez que o indivíduo contaminado é o grande disseminador 9 .

Quanto à distribuição de enteroparasitoses entre manipuladores de alimentos, verificouse freqüências variáveis para cada parasito. A observação do parasitismo por Giardia lamblia encontrada nesta pesquisa e com relação a alguns aspectos epidemiológicos permite concluir que a ocorrência desta protozoose foi bai$\mathrm{xa}$, pois os participantes apresentavam uma faixa etária com média de 18 a 55 anos. De fato, a literatura mostra que este parasito é encontrado principalmente em crianças com idade de 0 a 5 anos, e os adultos apresentam uma certa imunidade, não ocorrendo tantas reinfecções 10 .

Nota-se que o Blastocystis hominis foi o segundo protozoário de maior ocorrência. Esse protozoário, apesar de várias controvérsias e indefinições, tem assumido lugar de destaque em pacientes com AIDS, caracterizando como uma patologia emergente nos diversos países da América Latina 11,12,13. A alta freqüência desse microorganismo em manipuladores de alimentos pode ser justificada se for considerado que a principal forma de transmissão do mesmo é a via oral-fecal 14.

A falta de controle higiênico dos alimentos vendidos por pessoas que manipulam os mesmos, constitui uma das principais fontes de disseminação de enteropatógenos. Neste trabalho pode-se notar uma elevada ocorrência de Endolimax nana e Entamoeba coli. Embora estes organismos não sejam considerados patógenos, estes dados são alarmantes principalmente porque se trata de grupos de alto risco de transmissão, devido as atividades de manipulação de alimentos que realizam 15 .

Conclui-se que nesta cidade os manipuladores de alimentos apresentaram elevados índices de parasitoses. Os resultados desta pesquisa podem representar subsídios para futuras ações de controle das enteroparasitoses, bem como contribuir para o aprimoramento da educação sanitária destes profissionais, por meio de palestras educativas, baseadas na análise de risco potencial de contaminação dos alimentos. 


\section{Resumo}

Estudou-se a presença de enteroparasitas e os aspectos epidemiológicos em 238 indivíduos que trabalhavam em uma empresa de alimentos e trabalhadores de feiras livres e "sacolões", na cidade de Florianópolis, Santa Catarina, Brasil. As técnicas parasitológicas utilizadas neste estudo foram: Lutz, Baermann-Moraes, Graham e Faust, e as taxas de infecção parasitária encontradas, 42,85\% e 47,06\%, respectivamente. Os fatores sócio-econômicos, como distribuição de renda, escolaridade e categorias ocupacionais, foram importantes dentro deste contexto. O hábito de ingerir hortaliças e frutas foram os fatores mais associados ao alto grau de parasitismo. Conclui-se que, nesta cidade, os manipuladores de alimentos apresentaram elevados índices de parasitose, havendo necessidade de uma melhor vigilância epidemiológica por meio de exames parasitológicos e educação sanitária a todos esses indivíduos.

Manipulação de Alimentos; Infecções Parasitárias; Análise Parasitológica

\section{Referências}

1. Kãfertein EK, Motarjemi Y, Bettcher DW. Foodborne disease control: a transnational challenge. Emerg Infect Dis 1997; 3:503-10.

2. Fontes G, Oliveira KKL, Oliveira AKL, Rocha EMM. Influência do tratamento específico na prevalência de enteroparasitoses e esquistossomose mansônica em escolares do município de Barra de Santo Antônio, AL. Rev Soc Bras Med Trop 2003; 36:625-8.

3. Guedes MLS, Guedes JS. Bioestatística para profissionais da saúde. Rio de Janeiro: Ao Livro Técnico; 1988.

4. Guilherme ALF, Araújo SM, Falavigna DLM, Pupulim ART, Dias MLGG, Oliveira HS, et al. Prevalência de enteroparasitas em horticultores e hortaliças da Feira do Produtor de Maringá, Paraná. Rev Soc Bras Med Trop 1999; 32:405-11.

5. Grillo LP, Carvalho LR, Silva AC, Verreschi I, Sawaya AL. Influência das condições sócio-econômicas nas alterações nutricionais e na taxa de metabolismo de repouso em crianças escolares moradoras em favelas no município de São Paulo. Rev Assoc Med Bras 2000; 46:7-14.

6. Mulcahhy G, O’Neill S, Donnelly S, Dalton JP. Helminths at mucosal barriers - interaction with the immune system. Adv Drug Deliv Rev 2004; 56:853-68.

7. Hoton J. Global anthelmintic chemotherapy programs: learning from history. Trends Parasitol 2003; 19:405-9.

8. Fontes G, Oliveira KKL, Oliveira AKL, Rocha EMM. Influência do tratamento específico na prevalência de enteroparasitoses e esquistossomose mansônica em escolares do município de Barra de Santo Antônio, AL. Revista Soc Bras Med Trop 2003; 36:625-8.

\section{Colaboradores}

G. A. Cantos participou da orientação do projeto, da análise dos dados, da redação, da edição e da revisão do artigo final. A.C. Nolla contribuiu na revisão de literatura, na elaboração do projeto, na coleta e na análise de dados e na edição e redação do artigo final.
9. Carli GA. Parasitologia clínica. Seleção de métodos e técnicas de laboratório para o diagnóstico de parasitoses humanas. São Paulo: Atheneu; 2002.

10. Uchôa CMA, Lobo AGB, Bastos OMP, Matos AD. Parasitoses intestinais: prevalência em creches comunitárias da cidade de Niterói, Rio de Janeiro, Brasil. Rev Inst Adolfo Lutz 2001; 60:97-101.

11. Amato Neto V, Alarcón RSR, Gakiya E, Ferreira CS, Bezerra RC, Santos AG. Elevada porcentagem de balstocistose em escolares de São Paulo, SP. Rev Soc Bras Med Trop 2004; 37:354-6.

12. Amato Neto V, Alarcon RSR, Gakiya E, Bezerra RC, Ferreira CS, Braz LMA. Blatocistose: controvérsias e indefinições. Rev Soc Bras Med Trop 2003; 36: 515-7.

13. Cimerman S, Ladeira MCT, Iuliano WA. Blastocistose: nitazoxanida como nova opção terapêutica. Rev Soc Bras Med Trop 2003; 36:415-7.

14. Requena I, Hernández Y, Ramsay M., Salazar C, Devera R. Prevalencia de Blastocystis hominis en vendedores ambulantes de comida del municipio Caroní, Estado Bolívar, Venezuela. Cad Saúde Pública 2003; 19:1721-7.

15. Oliveira MC, Silva CV, Costa-Cruz JM. Intestinal parasites and commensals among individuals from a landless camping in the rural area of Uberlândia, Minas Gerais, Brazil. Rev Inst Med Trop S Paulo 2003; 45:173-6.

Recebido em 25/Jun/2004

Versão final reapresentada em 08/Out/2004 Aprovado em 14/Out/2004 